\title{
The Effectiveness of integrating spirituality into prenatal care on Improving Sleep Quality and Insomnia Severity among Pregnant Women: A Randomized Control Trial
}

\section{azam maleki ( $\nabla$ malekia@zums.ac.ir)}

Zanjan University of Medical Sciences https://orcid.org/0000-0001-7888-1985

\section{Nahid Moradi}

Zanjan University of Medical Sciences

\section{Saeedeh Zenoozian}

Zanjan University of Medical Sciences

\section{Research Article}

Keywords: Spiritual Counseling, Sleep Quality, Insomnia Severity, Pregnancy, Women's health

Posted Date: October 29th, 2021

DOI: https://doi.org/10.21203/rs.3.rs-1025124/v1

License: (c) (1) This work is licensed under a Creative Commons Attribution 4.0 International License. Read Full License 


\section{Abstract}

Background To determine the effectiveness of integrating spirituality into prenatal care on Improving Sleep Quality and Insomnia Severity among Pregnant Women. Methods This randomized controlled trial was carried out on 40 pregnant women recruited at five health centers of zanjan, Iran, 2020. The eligible women were allocated into two intervention and control groups according to the randomized design. Group counselling with spiritual content was carried out in eight sessions, two times a week at 16 to 20 weeks of gestation. The control group only received routine care. Data were collected using the Pittsburgh Sleep Quality Index (PSQI) and the Insomnia Severity Index (ISI) questionnaires in three stages, before the intervention, at 28 and 36 weeks of gestation. Statistical analysis was performed using the SPSS 16.0 software (SPSS Inc., Chicago, IL, USA). Results The results showed that comparing the mean score of insomnia severity, sleep quality and its components in the pre-intervention phase was not statistically significant between the two groups $(p>0.05)$. Comparison of the insomnia severity, sleep quality, and its components scores decreased statistically in the second and third trimesters compared to the first trimester in the intervention group than the control group $(\mathrm{p}<0.05)$. The effect of the intervention (Eta score) was $64 \%$. Conclusion The results showed that counselling with spiritual content could effectively ameliorate sleep quality and reduce insomnia severity in pregnant women. It seems that the approach is an acceptable basis to design intervention programs in this field that can be considered by midwives.

\section{Background}

One of the most common complaints during pregnancy is to have sleep disorders, such as insomnia, frequently waking up at night, high drowsiness during the day, mood swings, and unusual feelings during sleep(1). In one review, more than $80 \%$ of pregnant women had sleep disorders (2). According to the results of other studies, nearly half of pregnant women (45.7\%) suffer from poor sleep quality and $50 \%$ from insomnia $(1,3)$ A person's ability to fall asleep and the positive feeling after waking up indicate an ideal sleep quality(4). The quality varies in the different trimesters of pregnancy. So that, the prevalence of sleep disorders can be different from $63 \%$ in the first trimester to $80 \%$ in the second trimester (5). Sleep disorders during pregnancy can increase unfortunate consequences, such as preeclampsia, preterm labor, miscarriage, urgent cesarean delivery, gestational diabetes, postpartum depression, high levels of interleukin-6 (6-9).

To date, some studies have been conducted to improve sleep quality in pregnant women using a variety of pharmacological and non-pharmacological methods, each of which had different efficacy (10-12). Today, religious beliefs are considered by many researchers as major factors that affect physical and mental health and treat sleep disorders (13-16).

If spiritual health is at risk, the development of mental disorders, such as loneliness, anxiety, and loss of meaning in life will be likely. However, strengthening the spirituality in patients allows them to adapt effectively to their problems. Therefore, being connected with higher power and support from spiritual or religious sources can improve one's quality of life, control mental health disorders, and create 
interpersonal support (17). In some studies, the use of spiritual counselling has been emphasized in various aspects of pregnancy and childbirth, such as the stress during pregnancy and childbirth selfefficacy $(18,19)$. Spirituality is a key factor in promoting maternal and fetal health $(18-20)$; it plays an important role in the acceptance of pregnancy (21). According to studies, spiritual counselling has been effective in improving sleep quality for coronary artery disease patients (13), hemodialysis patients (14), women with breast cancer(15), and the elderly (16). Nevertheless, there is an information gap in the effectiveness of spiritual content counselling in improving sleep quality for women during pregnancy. Given the importance of culture and religion in many countries and the role of midwives in identifying the attitudes and spiritual beliefs of the individuals, the present study aimed to determine the effectiveness of spiritual content counseling on improving the sleep quality and insomnia severity of pregnant women.

\section{Methods}

\section{Setting and participants}

The present study was a randomized control trial to determine the effectiveness of counselling with spiritual content to improve prenatal sleep quality and to reduce sleep intensity in 2020 . The research setting included 5 health centers in Zanjan,Iran, city, and the research community included pregnant women referred to the centers. The sample size was calculated 16 people in each group based on the results of the similar study with considering $95 \%$ confidence interval, $80 \%$ power, the standard deviation of sleep quality 4.24 and 1.86 in two intervention and control groups respectively(22). Due to the possibility of a $10 \%$ attrition in sample size, it increased to 20 in each group. Thus, 40 eligible pregnant women were selected using the convenience sampling method and assigned to the two control and intervention groups via the simple random method. The inclusion criteria were the age group of 18 to 35 years, the gestational age of 20-16 weeks, willingness to participate in the study, scoring more than 5 based on The Pittsburgh Sleep Quality Index (PSQI), and scoring less than 22 based on the General Health Questionnaire (GHQ-28). The exclusion criteria were more than two absences from counselling sessions, consumption of sleeping pills for sleep control, medical and obstetrics complications during the study, withdrawal from the study, and participation in a similar training program (Chart 1 Participant flowchart).

\section{Procedure/ Intervention}

For the intervention group, spiritual content counselling was conducted based on the protocol of spiritual skills of Boulehri (23) and the study protocol of Asadi Zandi et al. (13). Accordingly, eight groups (groups of 6-8 people) were held for four following weeks. All the counselling sessions were attempted to use the principles and techniques of counselling to communicate effectively. The counselling session was full of respect and intimacy, high self-confidence to provide an incredible opportunity for individuals to participate in group discussions. The content of each counselling session is listed in Table 1. The counselling was conducted by a midwife that familiar with group counselling under the supervision of a clinical psychologist. 
Data were completed by participants using the personal information questionnaire, the Pittsburgh quality sleep questionnaire and the insomnia severity index questionnaire in three-stage, before the intervention, 28 and 36 weeks of gestational age.

\section{Individual information questionnaire}

The demographic questionnaire included age, level of education, employment status of the woman and her spouse, family income level, number of pregnancies, gestational age, number of abortions, pregnancy requirements, and residence status.

\section{The Pittsburgh Sleep Quality Index (PSQI)}

The Pittsburgh Quality Sleep Questionnaire was designed by Baysse, et al. and used to measure sleep quality over the past month. This includes the seven components: subjective sleep quality, sleep latency, sleep duration, habitual sleep efficiency, sleep disturbances, use of sleeping medication, and daytime dysfunction. The first four questions of this questionnaire are descriptive, and the questions of five to seventeen are multiple-choice. Scoring multiple-choice questions on a Likert scale ranges from zero to three, with no "zero scores" option, less than once a week "score one", once or twice a week "score two" and three More or less "points" are awarded during the week. Therefore, the total score range of the questionnaire varies from zero to 21. People who score five or less on the Pittsburgh Quality Sleep Questionnaire are considered to have a good sleep, and those who score more than five are considered undesirable. The reliability coefficient of the questionnaire in the study of Baysse et al. was 0.85 (24). In the study of Behroozifar et al., the reliability of the Persian version of the questionnaire was reported to be $0.74(25)$.

\section{Insomnia Severity Index (ISI)}

The insomnia severity Index is a brief self-report tool that assesses the patient's perception of the intensity of insomnia. This index has seven questions that evaluate the inability to start sleeping, inability to continue sleeping in the form of frequent waking up, waking up early, satisfaction with sleep patterns, daily dysfunction, significant insomnia problem for others, and anxiety (26). Scoring questions on 5 Likert scales ranges from zero (never) to 4 , so the score range is between 0 and 28 . The total score of the questionnaire is obtained by adding the score of the items. Higher scores indicate the high intensity of insomnia. A score of 15 to 21 also indicates moderate clinical insomnia, and 22 to 28 indicates severe insomnia. The reliability coefficient of this scale was reported in the study of Timpano et al., 0.85 (27), and the Persian version of the questionnaire in Yazdi et al .'s study was reported to be 0.80 (28).

\section{The General Health Questionnaire (GHQ-28)}

The 28- question form of the General Health Questionnaire (GHQ-28) was developed by Goldberg and Hiller in 1979. The questionnaire measures a person's general health in the last month (29) and includes four areas of physical complaints: anxiety, social maladaptation, and depression. GHQ-28 scoring is based on the patient's response from no way until more than usual, which is graded from zero to 3 . If the 
total score of each field is more than or equal to 14 , it indicates a disorder in that field, and if the total score of the questionnaire is more than 23 , it indicates a disorder in general health. The questionnaire has the desired validity and reliability (30). Cronbach's alpha coefficient of the Persian version of the questionnaire in the study of Taghavi et al. was reported to be 0.90 (31).

\section{Statistical methods}

Data were analyzed using SPSS software version 16 and ANCOVA, chi-square test, independent t-tests.

\section{Result}

\section{Baseline data}

The mean age of women in the intervention group was 26.65 years, in the control group was 28.55 years; the mean gestational age was 18.60 in the intervention group and 18.20 in the control group. Comparing the gestational age and age of women between the two groups was not statistically significant $(p>0.05)$. The results of Table 2 show that the comparison of individual profile variables in the two groups was not statistically significant, so the two groups were homogeneous( $p>0.05)$.

\section{Sleep Quality}

The results of Table 3 show that comparing the mean score of sleep quality and its components in the pre-intervention phase was not statistically significant $(p>0.05)$. Comparison of the overall score of sleep quality and its subjective sleep quality, sleep latency, sleep duration, sleep disturbances, use of sleeping medication, and daytime dysfunction components increased in the second and third trimesters compared to the first trimester in the control group and decreased in the intervention group. The observed differences between the two groups were statistically significant $(p<0.05)$. However, the score of the habitual sleep efficiency component did not change much in either group during the three trimesters and neither participant in the two groups used the drug to control sleep $(p>0.05)$. A comparison of the mean scores between the two groups in three time periods is shown in Figure 2.

\section{Insomnia Severity}

The results of Table 4 show that the mean score of insomnia severity in the pre-intervention phase was 7.05 in the control group and 7.05 in the intervention group. Comparing the mean insomnia severity in the pre-intervention phase was not statistically significant, so they were homogeneous( $p>0.05)$. In the control group, the comparison of the mean insomnia severity in the second and third trimesters increased compared to before the intervention and decreased in the intervention group. The observed differences between the two groups were statistically significant $(p<0.05)$. It was significant to compare the mean scores in the three time periods between the two groups $(p<0.05)$ (figure3). The effect size of intervention ("Eta" score) at the end of the follow-up period was about 0.66 , it shows that counselling with a spiritual content was associated with a $66 \%$ reduction in the severity of insomnia. 


\section{Discussion}

The results of the present study showed that counselling with a spiritual approach was effective in improving the sleep quality in pregnant women. In the intervention group, the mean score of sleep quality decreased in the second and third trimesters compared to the control group. Although no available studies were showing the effect of spiritual content counselling on sleep quality during pregnancy, the positive effects of spiritual counselling on improving sleep quality have been reported in the non-pregnant research community. Taheri et al. (2013) showed that after spiritual care based on the "Ghalb Salim" nursing model, the mean score of sleep quality in patients with coronary artery disease had a statistically significant difference in comparison with the control group (13). Soheili et al. (2013) in a similar study found that the score of disabled veterans' sleep quality after spirituality training based on the teachings of Islam was significantly reduced (32). In the present study, spiritual content counselling had a significant effect on all areas of sleep quality, except for habitual sleep efficiency. However, in the above studies, an improvement was observed in all areas of sleep quality.

Some studies have emphasized various beliefs about the biological and psychological effects of spiritual-oriented counselling $(33,34)$. Spirituality, as a powerful factor, can influence one's attitude, cognition, and behaviour, and as a mediator can affect one's thinking and evaluation process (35). In the Qur'an, God describes sleep with the word "Noum" and says in the ninth verse of Surah Naba "We have made your sleep a source of peace, the mentally and physically rejuvenation, and to rebuild the worn-out limbs and relieve any fatigue or discomfort". From the content of the tenth verse of Surah Anfal, it is clear that sleep causes the elimination of fear and the feeling of security. This shows that sleep and a sense of security complete each other(36).

The peace of mind caused by religious activities (e.g., listening to religious sounds) has been emphasized to reduce anxiety and pain during pregnancy $(37,38)$. Peace of mind and healthy sleep are essential in exuding positive hormones from the brain, improving protein metabolism, increasing the function of the immune system, and reducing the stress reactions in the body (34). According to our result, another positive effect of spirituality-based counselling is the improvement of sleep quality during pregnancy, from which the providers can benefit in the form of maternity care service packages.

In the present study, we used religious sounds during relaxation and meditation exercises, which had similar effects on the results of mindfulness and relaxation-based interventions (12). Mindfulness strategies play a role in reducing stress and regulating emotional response. For instance, yoga leads to communication with higher and superior powers. The results of the present study show that spiritual counselling as well as the two interventions listed are useful in reducing the cognitive and physical arousal associated with insomnia in pregnancy.

In the present study, part of the intervention focused on sleep health education and identifying sleep stimuli. It seems that increasing spiritual attention, along with health advice and behaviour modification, can have a positive effect on sleep quality. The present study was consistent with that of Lee et al. in 2017, and they showed that health behaviour education bases on the BETTER approach can be 
significantly reduced insomnia symptoms compared to the control group among 25 pregnant women who admitted to the hospital (39).

The results of the present study showed that after the intervention, the mean score of the areas of subjective sleep quality, sleep latency, sleep duration, sleep disturbances, use of sleeping medication, and daytime dysfunction in the intervention group decreased significantly. In contrast, the score of these areas in the control group had significant increase. Comparing the scores of habitual sleep efficiency in the two groups was not statistically significant. Also, none of the participants in either group used medication to control sleep disorders. In a clinical trial study, Felder et al. examined the efficacy of digital counselling using the cognitive-behavioural approach in treating the symptoms of insomnia among 208 pregnant women with a mean gestational age of 17 weeks. The intervention group received 6 sessions of online counselling per week in 5 areas (sleep restriction, stimulus control, cognitive therapy, relaxation techniques, and sleep hygiene training), and the control group received routine care. The follow-up period was 10 weeks after the end of the intervention. The results showed that the mean score of sleep quality and habitual sleep efficiency in the intervention group was significantly lower than the control group, while the mean score of sleep duration did not differ (40). The results of the above study were consistent with the results of the present study in terms of overall sleep quality score, but not in terms of habitual sleep efficiency and sleep duration, which can be due to easy access to an online consultant.

The results of a review of 24 articles show that the mean score of sleep quality during pregnancy was 6.07. About $45.7 \%$ of pregnant women experience some degree of diminished sleep quality during pregnancy. However, changes in sleep quality score increase in the third trimester were 1.68 times higher than in the second trimester (2). In the present study, the changes in the overall score of sleep quality in the control group were consistent with the results of the above study, so that the mean score of sleep quality of pregnant women increased from 9.26 in the first quarter to 11.47 in the third quarter. However, spiritual-oriented counselling reduced the overall score of sleep quality from 9.45 to 5.40 . Therefore, using the present approach can be considered as a basis for improving sleep quality during pregnancy.

Religious activities can be a social determining factor in the state of sleep. So those who engage in the religious activity are more likely to have a healthy sleep than those who do not. Based on the conceptual model proposed in a study, religious conflict may increase with hope, better support for mental health, a healthy lifestyle, reduced stress, healthier physiological function, limited substance use, and exposure to stress leading to healthy sleep (41). However, conflicting results have also been reported. For example, Gillum et al. (2013) showed that there was no significant relationship between participation in religious ceremonies in people over 40 years of age and sleep disorders (42), the results of which are inconsistent with the results of the present study because of the difference in the research population.

The results of the present study showed that spiritual content counselling was effective in improving the severity of insomnia in pregnant women. In the intervention group, the mean score of insomnia severity before the intervention decreased from 7.05 to 1.55 in the third trimester, and in the control group, it increased from 7.05 to 12.63 . Comparing the mean scores in the three time periods between the two 
groups was significant. So far, many interventions have been made to treat insomnia in pregnant women, but due to the adverse effects of drugs in pregnancy, researchers are trying to focus on nonpharmacological methods. The results of the present study are consistent with the results of some nonpharmacological interventions. As Jalal Marvi et al. showed (2019), distance education has positive effects on improving the intensity of insomnia in pregnant women (43). In another study, Felder et al. (2020) showed that the implementation of 6 sessions of digital counselling based on the cognitivebehavioural approach could alleviate the intensity of insomnia in pregnant women (40). Tomfohr-Madsen et al. reported similar results in 2017. In their study, holding 5 group counselling sessions based on the cognitive-behavioural approach had positive effects on alleviating the intensity of insomnia in pregnant women in the second trimester of pregnancy (44). The findings of our study showed that integration of sleep hygiene training with spiritual orders can be as effective as other interventions.

It can be argued that some cognitive patterns, psychological characteristics, and behavioural patterns created by spiritual-oriented methods lead to improved health and improved physiological function of the body, followed by high psychological resistance in inappropriate physical and social situations.

Accordingly, religious and spiritual practices lead to increased tolerance, patience, self-control, positivism, satisfaction, emotional control, optimism, self-efficacy (based on trust in God's blessing), altruism, kindness, and love(34). The skill of "spiritual resilience" is another technique of spiritual counselling that encourages the audience to overcome physical and mental problems in a variety of ways. The present study had several limitations. Data collection was performed using a self-report questionnaire and all participants were Muslim which should be considered in the generalizability of our findings.

\section{Implications for Practice and/or Policy}

In the present study, the effects of spiritual content counselling were not significant on habitual sleep efficiency. Given the importance of sleep in the physical and mental health of pregnant mothers, it is recommended that educational programs be conducted using other psychological approaches in this field.

\section{Conclusion}

The results of the present study showed that counselling with a spiritual content could effectively ameliorate sleep quality and reduce the insomnia severity in pregnant mothers. Also, improving sleep quality continued until the third trimester of pregnancy. However, the control group had worse sleep quality with increasing gestational age than the intervention group. It seems that the approach of the present study is an acceptable basis to design intervention programs in this field that can be considered by researchers, consultants, and midwives.

\section{List Of Abbreviations}

The Pittsburgh Sleep Quality Index (PSQI) 


\section{Declarations}

Ethical Approval: This article was part of MSc thesis and approved by the Ethics Committee of the Vice Chancellor for Research of Zanjan University of Medical Sciences, Iran, with the approval IR.ZUMS.REC.1398.153The objectives of the study were explained to the participating mothers and all participants signed written informed consent forms. Subjects could leave the study at any time, and they were also assured about the confidentiality of their data.

Funding Sources. This article was part of MSc thesis and funded by the Research Deputy of Zanjan University of Medical Sciences, Iran, with the approval number (The code "A-11-344-9").

Clinical Trial Registry and Registration number: The study was registered at the Iranian Registry of Clinical Trials under the IRCT20150731023423N15.

\section{Acknowledgements}

We would like to thank the Zanjan university's vice-chancellor of education and vice-chancellor of research and technology for their financial support to carry out the study (The code "A-11-344-9"). We would like to thank the staffs of the health centers of Abhar for their collaboration, and mothers for their participation

\section{Authorship}

This study was a part of MSc thesis of N.M.The conception, design of the study, and data collection process were undertaken by N.M. A.M was the supervisor and S.Z was adviser. They are also contributed to the design of the study, and reporting of the result. Analysis, interpretation and reporting were supervised by A.M and N.M. All authors contributed in drafting the article, revising it and in agreement with final version of the manuscript to be submitted to the journal and they also met the criteria of authorship

\section{References}

1. Sedov ID, Cameron EE, Madigan S, Tomfohr-Madsen LM. Sleep quality during pregnancy: A metaanalysis. Sleep medicine reviews. 2018;38:168-76.

2. Kaveh M. Sleep disorders in iranian pregnant women: A systematic review and meta-analysis. International Journal of Pharmaceutical and Biological Science Archive. 2019;7(6).

3. Kızılırmak A, Timur S, Kartal B. Insomnia in pregnancy and factors related to insomnia. The Scientific World Journal. 2012;2012.

4. Harvey AG, Stinson K, Whitaker KL, Moskovitz D, Virk H. The subjective meaning of sleep quality: a comparison of individuals with and without insomnia. Sleep. 2008;31(3):383-93. 
5. Lopes EA, Carvalho LB, Seguro PB, Mattar R, Silva AB, Prado LB, et al. Sleep disorders in pregnancy. Arquivos de neuro-psiquiatria. 2004;62(2a):217-21.

6. Okun ML, Coussons-Read ME. Sleep disruption during pregnancy: how does it influence serum cytokines? Journal of reproductive immunology. 2007;73(2):158-65.

7. Yang Z, Zhu Z, Wang C, Zhang F, Zeng H. Association between adverse perinatal outcomes and sleep disturbances during pregnancy: a systematic review and meta-analysis. The Journal of MaternalFetal \& Neonatal Medicine. 2020(just-accepted):1-261.

8. Dixon CL. Sleep Deprivation \& Pregnancy Related Risks. International Journal of Childbirth Education. 2014;29(3).

9. Won $\mathrm{CH}$. Sleeping for two: the great paradox of sleep in pregnancy. Journal of Clinical Sleep Medicine. 2015;11(06):593-4.

10. Shojaei Sorak S, Asgarizadeh Q, Mousavinasab MH. The Effectiveness of Relaxation Training on General Health and Sleep Quality of Pregnant Women in the Last Trimester of Pregnancy. Journal of Shahid Sadoughi University of Medical Sciences. 2017;24(11):887-98.

11. Liu YH, Lee CS, Yu CH, Chen CH. Effects of music listening on stress, anxiety, and sleep quality for sleep-disturbed pregnant women. Women \& health. 2016;56(3):296-311.

12. Bacaro V, Benz F, Pappaccogli A, De Bartolo P, Johann AF, Palagini L, et al. Interventions for sleep problems during pregnancy: a systematic review. Sleep Medicine Reviews. 2019:101234.

13. Taheri ZS, Zandi MA, Ebadi A. The effect of spiritual care based on Ghalbe Salim Model on the sleep quality of the patients with coronary artery disease. Iran J Crit Care Nurs. 2014;7(2):92-101.

14. Yang JY, Huang JW, Kao TW, Peng YS, Lu CS, Chen DL, et al. Impact of spiritual and religious activity on quality of sleep in hemodialysis patients. Blood purification. 2008;26(3):221-5.

15. Sajadi M, Niazi N, Khosravi S, Yaghobi A, Rezaei M, Koenig HG. Effect of spiritual counseling on spiritual well-being in Iranian women with cancer: A randomized clinical trial. Complementary therapies in clinical practice. 2018;30:79-84.

16. Ghadampour E RS, Rezaeifar N. Measuring the Effectiveness of Group Spirituality Therapy on Sleep Quality and Mental Toughness in Elderly Subjects in Khorramabad. Journal of Gerontology (joge). 2018;3(2):31-9.

17. Koenig HG. Research on religion, spirituality, and mental health: a review. Canadian journal of psychiatry Revue canadienne de psychiatrie. 2009;54(5):283-91.

18. Haghighat M, Mirghafourvand M, Mohammad-Alizadeh-Charandabi S, Malakouti J, Erfani M. The effect of spiritual counseling on stress and anxiety in pregnancy: A randomized controlled clinical trial. Iranian Red Crescent Medical Journal. 2018;20(4).

19. Mohamadirizi S, Mohamadirizi M, Mohamadirizi S, Mahmoodi F. The effect of religious-spiritual support on childbirth self-efficacy. Journal of education and health promotion. 2018;7:14.

20. Tayebi S, Montaseri Z, Edraki M, Akbarzadeh M. Relationship between Mothers' Spiritual Health Scores with Newborns' Physical Development Indices and Physiologic Parameters in Hazrat Zeinab 
Training Hospital. Iranian Journal of Neonatology IJN. 2020;11(1):51-9.

21. Rodriguez AL, Arcara J, Deardorff J, Gomez AM. The association between religiosity and pregnancy acceptability among Latino/a young adults: does generational status matter? Culture, Health \& Sexuality. 2020;22(2):184-200.

22. Bolhari M. Spiritual Skills Training. Mirzaie M, Ghaempanah Z, editors2013.

23. Buysse DJ, Reynolds CF, 3rd, Monk TH, Berman SR, Kupfer DJ. The Pittsburgh Sleep Quality Index: a new instrument for psychiatric practice and research. Psychiatry research. 1989;28(2):193-213.

24. Behrouzifar S, Zenouzi S, Nezafati M, Esmaeili H. Possible Effective Factors on the Sleep Quality and Quantity of Patients after Coronary Artery Bypass Graft. Journal of Ilam University of Medical Sciences. 2008;16(3):59-70.

25. Morin CM, Bastien C, Guay B, Radouco-Thomas M, Leblanc J, Vallieres A. Randomized clinical trial of supervised tapering and cognitive behavior therapy to facilitate benzodiazepine discontinuation in older adults with chronic insomnia. The American journal of psychiatry. 2004;161(2):332-42.

26. Timpano KR, Carbonella JY, Bernert RA, Schmidt NB. Obsessive compulsive symptoms and sleep difficulties: exploring the unique relationship between insomnia and obsessions. Journal of psychiatric research. 2014;57:101-7.

27. Yazdi Z, Sadeghniiat-Haghighi K, Zohal MA, Elmizadeh K. Validity and reliability of the Iranian version of the insomnia severity index. The Malaysian journal of medical sciences: MJMS. 2012;19(4):31-6.

28. Noorbala A, Yazdi SB, Yasamy M, Mohammad K. Mental health survey of the adult population in Iran. The British Journal of Psychiatry. 2004;184(1):70-3.

29. Noorbala A, Mohammad K. The validation of general health questionnaire-28 as a psychiatric screening tool. Hakim Research Journal. 2009;11(4):47-53.

30. Taghavi S. Validity and reliability of the general health questionnaire (ghq-28) in college students of shiraz university. Journal of psychology. 2002;5(4):381-98.

31. Soheyli M, Rahimi A, Chavoshi A. Effect of Sleep Based on Islamic Pattern on the Quality of Sleep in Mustard Gas Chemical Casualties. Quarterly of Iranian Journal of War \& Public Health. 2014;6(4):151-6.

32. Seybold KS. Biology of Spirituality. Perspectives on Science \& Christian Faith. 2010;62(2).

33. Srivastava A, Krishna A. Psycho-Physiological Mechanism of Spirituality-Health Relationship: Theoretical Conceptualisation. J Indian Health Psychol.2(1):1-9.

34. Asad zandi M. Analysis of Nursing Concepts and Theories (along with the presentation of the Salim Heart Model). Tehran: Heidary 287-300; 2014

35. Wnuk M, Marcinkowski JT. Do existential variables mediate between religious-spiritual facets of functionality and psychological wellbeing. Journal of religion and health. 2014;53(1):56-67.

36. Sri W, Tutik R, Hernandia D, Apriliyani YW, Siti DN. Spiritual Intervention and Thermal Stimulation in Pregnant Women with Back Pain. Indian Journal of Public Health Research \& Development. 
2019;10(8):2746-50.

37. Khodakarami B, Golalizadeh Bibalan F, Soltani F, Soltanian A, Mohagheghi H. Impact of a counseling program on depression, anxiety, stress, and spiritual intelligence in pregnant women. Journal of Midwifery and Reproductive Health. 2017;5(2):858-66.

38. Lee KA, Gay CL. Improving sleep for hospitalized antepartum patients: a non-randomized controlled pilot study. Journal of Clinical Sleep Medicine. 2017;13(12):1445-53.

39. Felder JN, Epel ES, Neuhaus J, Krystal AD, Prather AA. Efficacy of Digital Cognitive Behavioral Therapy for the Treatment of Insomnia Symptoms Among Pregnant Women: A Randomized Clinical Trial. JAMA psychiatry. 2020.

40. Hill TD, Deangelis R, Ellison CG. Religious involvement as a social determinant of sleep: an initial review and conceptual model. Sleep health. 2018;4(4):325-30.

41. RF Gillum MD M. Religious behavior, sleep quantity, sleep quality, and sleep disorders in American adults. The journal of behavioral health services \& research. 2013;40(1):133.

42. Jalal Marvi F, Kordi M, Mazlom SR, Rezaei Talab F. Comparing the Effect of Training Based on Continuous Care Model and Telehealth on Severity of Insomnia in Pregnant Women. Journal of North Khorasan University of Medical Sciences. 2019;11(3):38-45.

43. Tomfohr-Madsen LM, Clayborne ZM, Rouleau CR, Campbell TS. Sleeping for two: an open-pilot study of cognitive behavioral therapy for insomnia in pregnancy. Behavioral sleep medicine. 2017;15(5):377-93.

44. Hatami A, Badrani MR, Kamboo MS, Jahangirimehr A, Hemmatipour A. An investigation of the relationship of spiritual intelligence and resilience with attitude to fear of childbirth in pregnant women. Journal of Evolution of Medical and Dental Sciences-Jemds. 2019;8(1):5.

\section{Tables}

Table 1: Description of the content of spiritual counseling sessions 


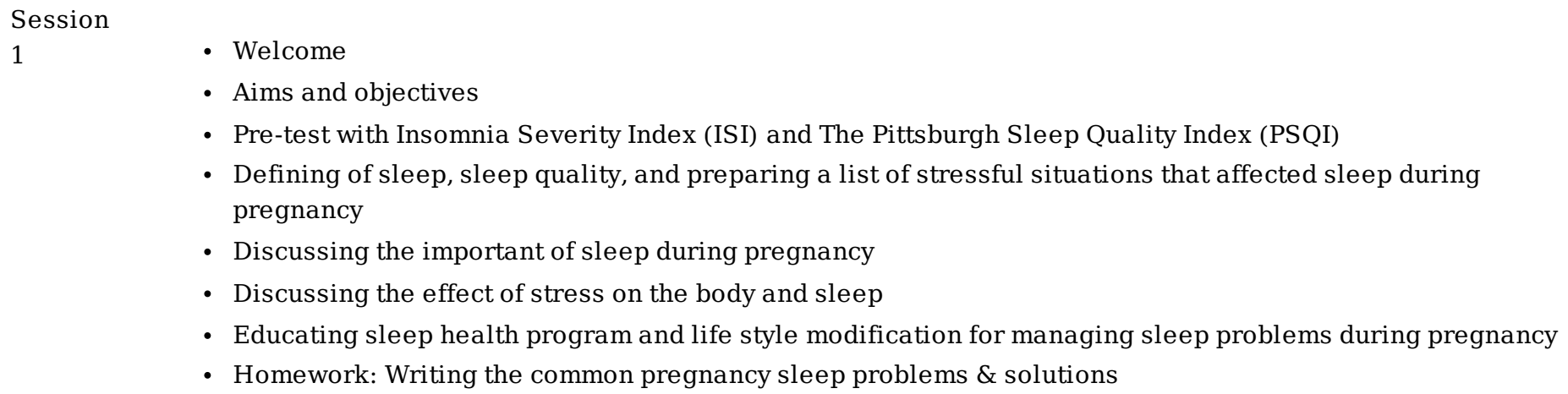

Session

- Encouraging to express their common pregnancy sleep problems \& reviewing the solutions

- Discussing one's attitude towards spiritual issues, the role of God and religion in her life

- Discussing one's experiences of reading the Qur'an and listening to the sound of the Qur'an in her calming and the soul

- Homework: Writing the spiritual aspects of the pregnancy

Session

3

4

Session

Session

5

Session

6

Session

7
- Talking about the spiritual aspects of pregnancy, childbearing, and God's reward for pregnant women

- Reciting of verses and hadiths about sleep and the role of sleep comfortably in peace of mind

- Creating a spiritual environment with listening to the voice of the Quran in Surah Ar-Rahman (with the voice of Abdul Basit ) for 5 min

- Discussing of one's feels after listening to the voice of the Quran

- Homework: Listening to the voice of the Quran and relax their muscles with deep breathing for getting rid of the stress / twice daily for 10 to 15 minutes

- Reviewing the content of previous sessions and summarize members' opinions

- Blessings of God and the role of it in reinterpreting concept of pregnancy and overcoming the worrisome symptoms of pregnancy

- Discussing of prayer therapy to reduce the worries, get rid of some symptoms of pregnancy and to increase hope, resilience

- Homework: Encouraging to create a daily spiritual space of time or place at home

- Writing the experience of participating in religious programs or doing spiritual issues

- Discussing one's experiences of participating in religious programs or doing spiritual issues

- Discussing on the role of charity, trust, recourse, patience, kindness and forgiveness in managing emotions and life stressors

- Discussing on the role of gratitude to God in enduring hardships, increasing hope and self-confidence, adapting and accepting the situation

- Encouraging to strengthen one's inner hope, locus of control, and resilience

- Reciting the verses of Surah Maryam with the voices of the volunteer members with focusing its meaning

- Homework: Writing down situations that make you feel guilty or angry about yourself or others

- Discussing on one's experiences of forgiveness in life and anger towards the guilty

- Discussing the meaning of life with the birth of a baby

- Listening to the "Nature's Music" the sound of birds, rivers and waterfalls with illustration hugging her baby

- Encouraging to participate in charity, kindness and forgiveness

- Homework: Writing some strategies to cope with stress and improving sleep quality
- Reviewing their strategies to cope with stress and improving sleep quality 
- Designing a play of a stressful situation, charting a table of tensions, the severity of tension, behavior and attitudes, satisfaction with the coping method employed in those situations, and simple suggestions to cope with stress

- Homework: Listening to the voice of the Quran and relax their muscles with deep breathing for getting rid of the stress / twice daily for 10 to 15 minutes

Session

8

- Reviewing the content of previous sessions and summarize members' opinions

- Discussing one's experiences of spiritual self-awareness, problem-solving skills with a spiritual approach

Table 2: Comparison of the frequency distribution of demographic characteristics between two study groups

\begin{tabular}{|c|c|c|c|c|c|c|}
\hline \multicolumn{2}{|c|}{ Social and Midwifery characteristics } & \multicolumn{2}{|c|}{ Intervention } & \multicolumn{2}{|c|}{ Control } & \multirow[t]{2}{*}{$P$ value } \\
\hline & & $\mathrm{N}$ & $\%$ & $\mathrm{~N}$ & $\%$ & \\
\hline \multirow[t]{3}{*}{ Education } & Guidance & 3 & 15 & 7 & 35 & \multirow{3}{*}{0.31} \\
\hline & Diploma & 13 & 65 & 9 & 45 & \\
\hline & Academic & 4 & 20 & 4 & 20 & \\
\hline \multirow[t]{2}{*}{ Job } & Unemployed & 20 & 100 & 19 & 95 & \multirow[b]{2}{*}{0.50} \\
\hline & Employed & 0 & 0 & 1 & 5 & \\
\hline \multirow[t]{3}{*}{ Family monthly income } & Not enough & 2 & 10 & 1 & 5 & \multirow{3}{*}{0.42} \\
\hline & To some degree enough & 7 & 35 & 11 & 55 & \\
\hline & Sufficient & 11 & 55 & 8 & 40 & \\
\hline \multirow[t]{2}{*}{ Housing status } & Owner & 8 & 40 & 7 & 35 & \multirow[b]{2}{*}{0.74} \\
\hline & Rented & 12 & 60 & 13 & 65 & \\
\hline \multirow[t]{2}{*}{ Gravid } & First pregnancy & 10 & 50 & 10 & 50 & \multirow[t]{2}{*}{1} \\
\hline & $\geq$ two pregnancy & 10 & 50 & 10 & 50 & \\
\hline
\end{tabular}

Table3. The comparison of sleep quality between the two groups in three stage (pre intervention, 28 and 36 weeks of gestation) 


\begin{tabular}{|c|c|c|c|c|c|c|c|c|c|c|}
\hline \multirow[t]{2}{*}{ Variables } & \multirow[t]{2}{*}{ Time } & \multicolumn{2}{|c|}{ Intervention } & \multicolumn{2}{|l|}{ Control } & \multirow{2}{*}{$\begin{array}{l}* * \mathrm{P} \\
\text { Value }\end{array}$} & \multirow[t]{2}{*}{$\mathrm{F}$} & \multirow[t]{2}{*}{ Eta } & \multirow{2}{*}{$\begin{array}{l}\text { Mean } \\
\text { diff }\end{array}$} & \multirow{2}{*}{$\begin{array}{l}* \mathrm{P} \\
\text { Value }\end{array}$} \\
\hline & & Mean & SD & Mean & SD & & & & & \\
\hline Sleep quality & Baseline & 9.45 & 2.30 & 9.26 & 2.15 & 0.75 & & & & \\
\hline \multirow[t]{2}{*}{ Total score } & $28^{\text {th }} \mathrm{w}$ & 5.70 & 1.55 & 11.63 & 2.06 & 0.001 & 66.90 & 0.64 & -3.93 & 0.001 \\
\hline & $36^{\text {th }} \mathrm{w}$ & 5.40 & 1.56 & 11.47 & 1.54 & 0.001 & & & & \\
\hline \multirow{3}{*}{$\begin{array}{l}\text { Subjective sleep } \\
\text { quality }\end{array}$} & Baseline & 0.8 & 0.69 & 1.00 & 0.66 & 0.36 & & & & \\
\hline & $28^{\text {th }} w$ & 0.3 & 0.47 & 1.58 & 0.69 & 0.001 & 19.90 & 0.35 & -0.96 & 0.001 \\
\hline & $36^{\text {th }} \mathrm{w}$ & 0.1 & 0.30 & 1.53 & 0.69 & 0.001 & & & & \\
\hline \multirow[t]{3}{*}{ Sleep latency } & Baseline & 1.75 & 0.63 & 1.57 & 0.60 & 0.39 & & & & \\
\hline & $28^{\text {th }} \mathrm{w}$ & 0.85 & 0.58 & 2.05 & 0.62 & 0.001 & 37.49 & 0.50 & -0.79 & 0.001 \\
\hline & $36^{\text {th }} w$ & 0.80 & 0.41 & 2.15 & 0.60 & 0.001 & & & & \\
\hline \multirow[t]{3}{*}{ Sleep duration } & Baseline & 0.45 & 0.82 & 0.52 & 0.84 & 0.77 & & & & \\
\hline & $28^{\text {th }} \mathrm{w}$ & 0.1 & 0.30 & 0.73 & 0.87 & 0.001 & 7.09 & 0.16 & -0.51 & 0.002 \\
\hline & $36^{\text {th }} \mathrm{w}$ & 0 & 0 & 0.84 & 0.83 & 0.001 & & & & \\
\hline \multirow[t]{3}{*}{ Sleep efficiency } & Baseline & 3.0 & 0.01 & 2.94 & 0.22 & 0.31 & & & & \\
\hline & $28^{\text {th }} \mathrm{w}$ & 3.0 & 0.01 & 2.89 & 0.45 & 0.31 & 1.05 & 0.02 & 0.08 & 0.31 \\
\hline & $36^{\text {th }} w$ & 3.0 & 0.01 & 2.89 & 0.45 & 0.31 & & & & \\
\hline \multirow[t]{3}{*}{ Sleep disturbances } & Baseline & 0.90 & 0.30 & 0.89 & 0.31 & 0.95 & & & & \\
\hline & $28^{\text {th }} \mathrm{w}$ & 0.55 & 0.51 & 0.94 & 0.22 & 0.004 & 6.07 & 0.14 & -0.26 & 0.004 \\
\hline & $36^{\text {th }} \mathrm{w}$ & 0.60 & 0.50 & 1.0 & 0.01 & 0.001 & & & & \\
\hline \multirow[t]{3}{*}{ Daytime dysfunction } & Baseline & 0.7 & 0.57 & 0.78 & 0.71 & 0.66 & S & & & \\
\hline & $28^{\text {th }} \mathrm{w}$ & 0.3 & 0.47 & 1.57 & 0.6 & 0.001 & 24.46 & 0.41 & -0.79 & 0.001 \\
\hline & $36^{\text {th }} w$ & 0.2 & 0.41 & 1.21 & 0.53 & 0.001 & & & & \\
\hline \multirow[t]{3}{*}{ Sleeping medication } & Baseline & 0 & 0 & 0 & 0 & 0 & & & & \\
\hline & $28^{\text {th }} \mathrm{w}$ & 0 & 0 & 0 & 0 & 0 & 0 & 0 & 0 & 0 \\
\hline & $36^{\text {th }} w$ & 0 & 0 & 0 & 0 & 0 & & & & \\
\hline
\end{tabular}


Table4. The comparison of insomnia severity between the two groups in three stage (pre intervention, 28 and 36 weeks of gestation)

\begin{tabular}{|c|c|c|c|c|c|c|c|c|c|c|}
\hline \multirow[t]{2}{*}{ Variables } & \multirow[t]{2}{*}{ Time } & \multicolumn{2}{|c|}{ Intervention } & \multicolumn{2}{|c|}{ Control } & \multirow[t]{2}{*}{$* * \mathrm{P}$ Value } & \multirow[t]{2}{*}{$\mathrm{F}$} & \multirow[t]{2}{*}{ Eta } & \multirow{2}{*}{$\begin{array}{l}\text { Mean } \\
\text { diff }\end{array}$} & \multirow[t]{2}{*}{ *P Value } \\
\hline & & Mean & SD & Mean & $\mathrm{SD}$ & & & & & \\
\hline \multirow[t]{3}{*}{ Insomnia severity } & Baseline & 7.05 & 4.29 & 7.05 & 4.19 & 0.99 & \multirow{3}{*}{8.79} & \multirow{3}{*}{0.66} & \multirow{3}{*}{-6.66} & \multirow{3}{*}{0.001} \\
\hline & $28^{\text {th }} w$ & 2.55 & 1.60 & 11.47 & 3.23 & 0.001 & & & & \\
\hline & $36^{\text {th }} w$ & 1.55 & 0.94 & 12.63 & 3.7 & 0.001 & & & & \\
\hline
\end{tabular}

\section{Figures}




\section{CONSORT Flow Diagram}

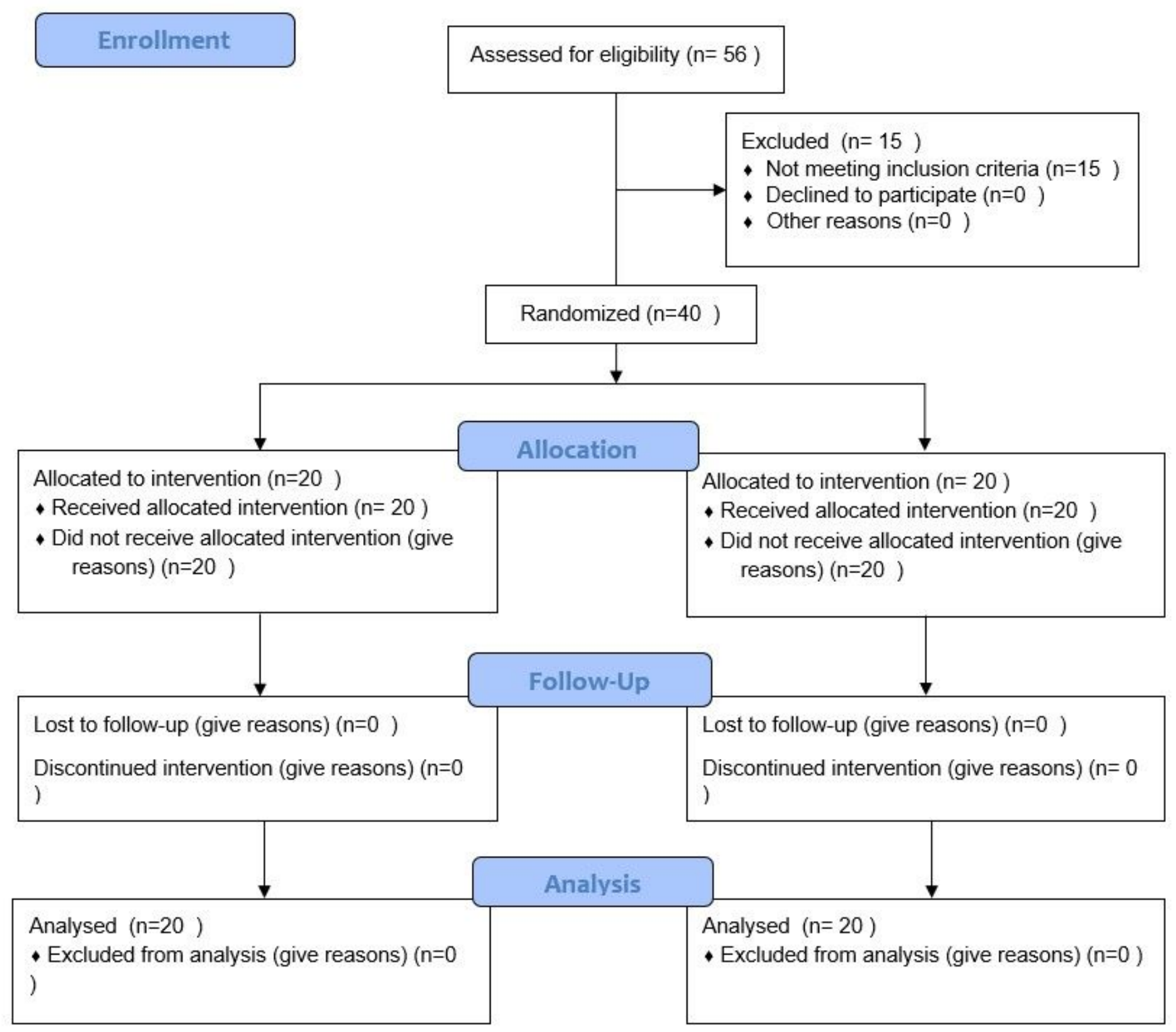

\section{Figure 1}

Chart of participant's enrollment and procedure 


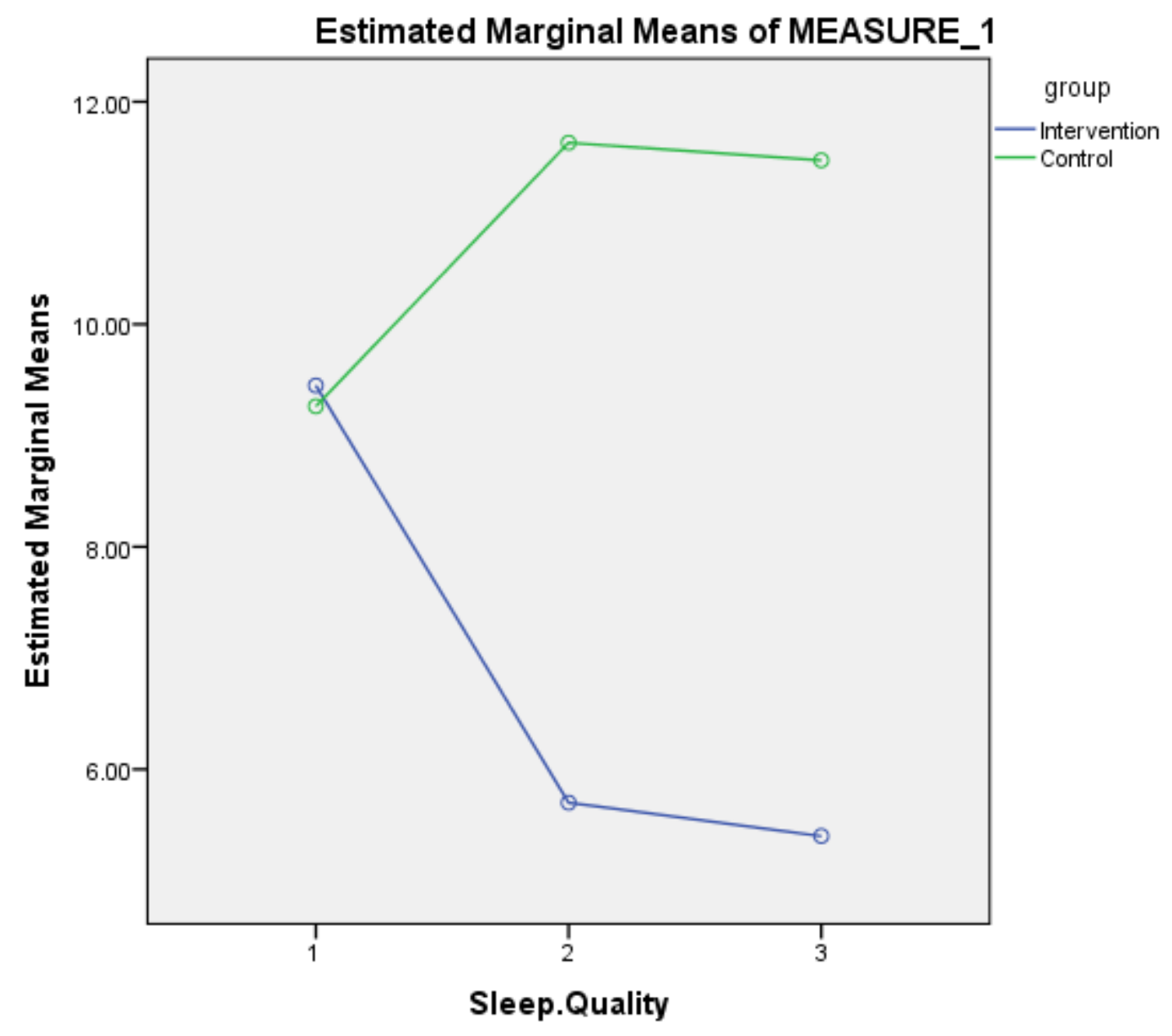

Figure 2

Chart of Insomnia severity index (ISI) in two groups 